BNL-77070-2006-CP

\title{
Fiddling Carbon Strings with Polarized Proton Beams
}

\author{
Haixin Huang (BNL), Kazuyoshi Kurita (RIKEN)
}

Presented at the BIW06 - Beam Instrumentation Workshop 2006

Batavia, Illinois

May 1-4, 2006

May 2006

Collider-Accelerator Department

Brookhaven National Laboratory

P.O. Box 5000

Upton, NY 11973-5000

www.bnl.gov

Notice: This manuscript has been authored by employees of Brookhaven Science Associates, LLC under Contract No. DE-AC02-98CH10886 with the U.S. Department of Energy. The publisher by accepting the manuscript for publication acknowledges that the United States Government retains a non-exclusive, paid-up, irrevocable, world-wide license to publish or reproduce the published form of this manuscript, or allow others to do so, for United States Government purposes. 


\section{DISCLAIMER}

This report was prepared as an account of work sponsored by an agency of the United States Government. Neither the United States Government nor any agency thereof, nor any of their employees, nor any of their contractors, subcontractors, or their employees, makes any warranty, express or implied, or assumes any legal liability or responsibility for the accuracy, completeness, or any third party's use or the results of such use of any information, apparatus, product, or process disclosed, or represents that its use would not infringe privately owned rights. Reference herein to any specific commercial product, process, or service by trade name, trademark, manufacturer, or otherwise, does not necessarily constitute or imply its endorsement, recommendation, or favoring by the United States Government or any agency thereof or its contractors or subcontractors. The views and opinions of authors expressed herein do not necessarily state or reflect those of the United States Government or any agency thereof. 


\title{
Fiddling Carbon Strings with Polarized Proton Beams
}

\author{
Haixin Huang ${ }^{\dagger}$ and Kazuyoshi Kurita* $\mathbb{1}$ \\ ${ }^{\dagger}$ Collider Accelerator Division, Brookhaven National Laboratory, Bldg. 902, Upton, New York 11973 , \\ USA \\ * Department of Physics, Rikkyo University, Nishi-Ikebukuro Toshima, Tokyo, 171-8501, Japan \\ "RI Beam Science Laboratory, RIKEN, Wako, Saitama, 351-0198, Japan
}

\begin{abstract}
An innovative polarimeter based on proton carbon elastic scattering in the Coulomb Nuclear Interference (CNI) region was first tested in the Brookhaven AGS successfully. CNI Polarimeters were then installed in the AGS and both RHIC rings. The polarimeter consists of ultra-thin carbon targets and silicon strip detectors. The waveform digitizers are used for signal readout, which allows deadtime-less data processing on the fly. Polarimeters are crucial instrumentation for the RHIC spin physics program. This paper summarizes the polarimeter design issues and operation results.
\end{abstract}

Keywords: proton beams, accelerator instrumentation.

PACS: 25.40.Ve, 13.88.+e, 13.85.Dz, 29.27.Hj

\section{INTRODUCTION}

The collision of polarized proton beams at RHIC will provide qualitatively new an exciting physics. The RHIC spin project collides up to $250 \mathrm{GeV}$ polarized proton beams and will open up the unique physics opportunity of studying spin effects in hard processes at high luminosities, including the measurement of the gluon polarization and the quark and anti-quark polarization by flavor. It will allow the study of the spin structure of the proton and also the verification of the well-documented expectations of the spin effects in perturbative QCD and parity violation I W and $\mathrm{Z}$ production. Development of a good polarimeter, which works at the proton beam energy up to $250 \mathrm{GeV}$, is of vital importance for the RHIC spin program.

Figure 1 shows a schematic view of RHIC accelerator complex. RHIC is the first and the only polarized collider in the world. The polarized $\mathrm{H}^{-}$beam from the optically pumped polarized ion source is accelerated through radio frequency quadrupole and the $200 \mathrm{MeV}$ linac. The beam polarization measured by the $200 \mathrm{MeV}$ polarimeter is about $80 \%$. The beam is then strip-injected and accelerated in the AGS Booster up to $1.5 \mathrm{GeV}$ kinetic energy. Only one bunch of the twelve RF buckets in the AGS is filled, and the polarized proton beam intensity can be as high as $2.2 \times 10^{11}$ proton per 
fill. In the AGS, the polarized proton beam is accelerated up to $24.3 \mathrm{GeV}$, passing through numerous spin depolarizing resonances. Two partial snakes separated by $1 / 3$ of the ring are used to maintain polarization. The beam is then injected through the AtR transfer line into RHIC rings. Two full snakes in each ring are sued to maintain polarization in RHIC. It requires careful accelerator tuning and polarimeters provide the most important parameter, namely the beam polarization.

\section{Polarization}

It is known that protons have so called "spin". Spin is a quantum mechanical quantity but can be considered as "a small magnet" as a classical analogy. Therefore, protons cannot be considered as a spherically symmetric particle but to have an orientation. In three-dimensional space, protons can point to any direction.

A group of protons can have an orientation as a whole. For example, protons in free ideal gas point to random direction and the orientation of the group averages out. In this case, protons are considered to have "no polarization" or have $0 \%$ polarization. When all the protons point to one direction, on the contrary, protons are considered to be fully polarized or have $100 \%$ polarization. In reality what we can obtain is a polarization in between the two extreme cases described above. Quantitatively the polarization $P$ is defined as

$$
P=\frac{N_{\uparrow}-N_{\downarrow}}{N_{\uparrow}+N_{\downarrow}}
$$

where $N_{\uparrow}$ and $N_{\downarrow}$ are the number of protons pointing up and down, respectively.

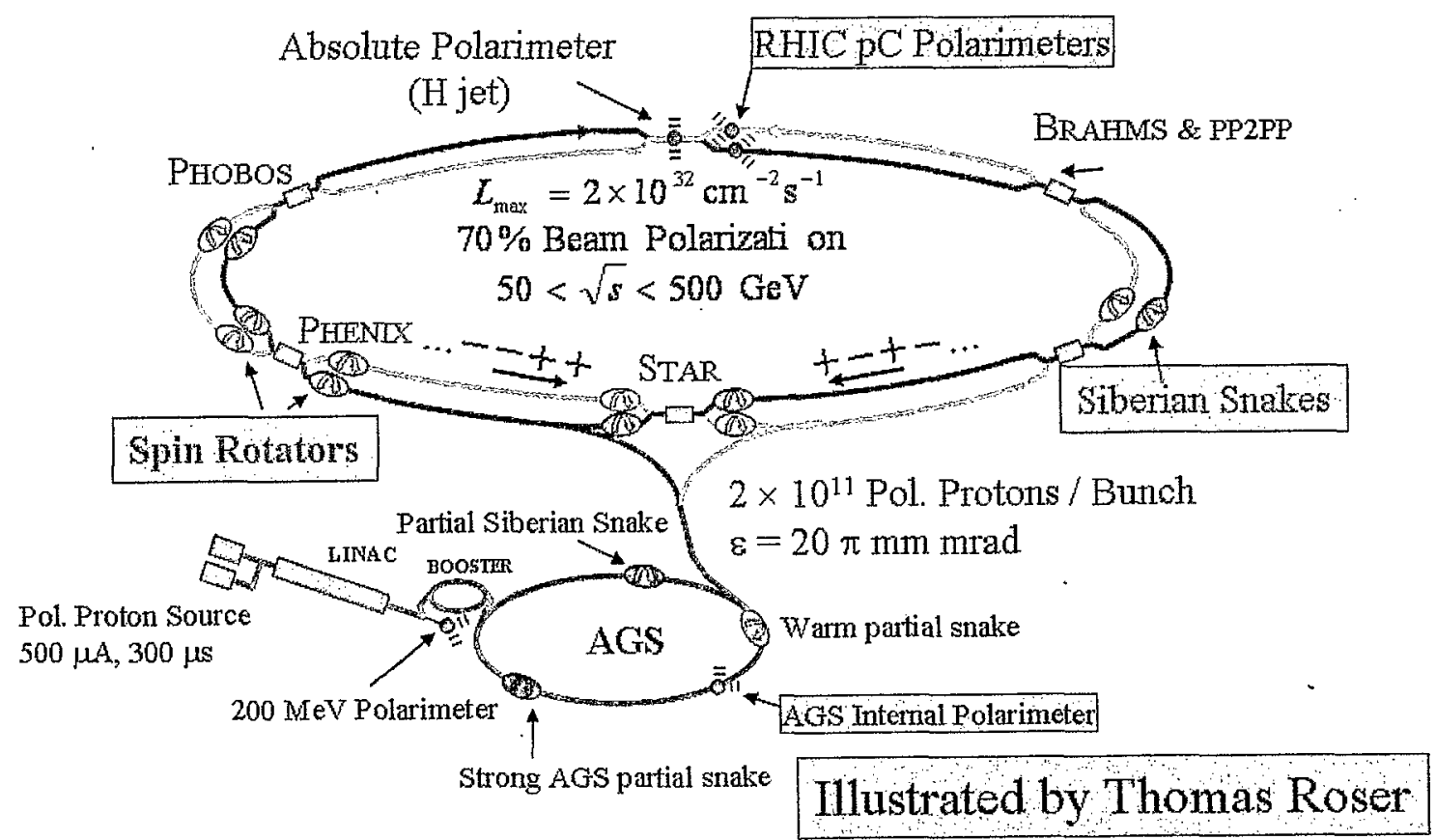

FIGURE 1. Schematic view of RHIC polarized proton collider. 


\section{How to Measure Polarization}

Next step is how to measure the proton polarization. There are elementary physical processes, which are known to have spin dependences. Assume the proton beam is $100 \%$ polarized in vertical direction. After hitting a fixed target, some beam particles are scattered left and some are scattered right. The asymmetry observed in the reaction $A_{N}$ is defined as

$$
A_{N}=\frac{N_{L}-N_{R}}{N_{L}+N_{R}}
$$

where $N_{L}$ and $N_{R}$ are the number of beam particles which are scattered left and right respectively. $A_{N}$ is called "analyzing power" and determines the inherent sensitivity of the polarimeter.

\section{Choice of Polarimeter}

There were possible physics processes, which can be applicable for the RHIC polarimeter. Proton-proton elastic scattering, inclusive pion productions and elastic scattering in the Coulomb-Nuclear Interference (CNI) region are the three processes that are taken into consideration.

First of all, the most commonly used proton-proton elastic scattering was considered. However, $A_{N}$ of proton-proton elastic scattering is known to decrease with increasing beam momentum and the $A_{N}$ is expected to be too small at the RHIC top energy.

Inclusive pion productions are known to have large $A_{N}$ at beam energy of $200 \mathrm{GeV}$ from FNAL-E704. The original proposal for the RHIC polarimeter was based on this process. However, due to the limitation of the detector space in the RHIC tunnel and the high cost for a magnetic spectrometer, the development of this polarimeter was discontinued.

Small angle elastic scattering of hadrons in the CNI region has long been advocated for polarimetry. The predicted asymmetry is significant and largely independent of energy for energy above a few $\mathrm{GeV}$. The prediction rests on hadronic spin flip being small, which is expected for high energies. The CNI process has been proposed for RHIC polarimetry using a hydrogen jet target and in collider mode using the pp2pp experiment. Both would be $p p$ CNI. It is also possible to use a carbon target, $p C$ CNI, which is simpler and cheaper than a hydrogen jet, and can be installed in the individual rings, vs. requiring collision of both rings as for the pp2pp experiment. In addition, carbon target is easier to handle in the vacuum. The analyzing power $A_{N}$ for $p C \mathrm{CNI}$ is similar to $p p \mathrm{CNI}$ (both about 0.04 ) and the cross section $\sigma$ is high, giving a very large figure of merit $\sigma A_{N}{ }^{2}$. However, for $p C$ CNI, the proton scattered forward is not easily detectable (it stays within the beam), and the energy of the recoil carbon nucleus is $100-1000 \mathrm{keV}$. The low energy carbon would stop in most targets. The very thin ribbon carbon targets developed at $\mathrm{IUCF}^{1}$ is crucial to the $p C$ CNI polarimeter: both for survival in the RHIC beam and to get the carbon nuclei out of the target in the $\mathrm{CNI}$ region where the recoil carbon carries only hundreds of keV kinetic energy. 
Due to the simplicity of the detector system, the cost and development time of pcarbon CNI polarimeter are significantly less than other polarimeter options. It is expected that the relative beam polarization can be determined by using a $p C \mathrm{CNI}$ polarimeter to $10 \%$ for each measurement in seconds at full luminosity. The polarimeter can be calibrated using a polarized proton jet target later to $5 \%$.

Elastic scattering in the small angle CNI region is predicted ${ }^{2}$ to have a calculable analyzing power of about 3-5\% as well as a large cross section over the whole RHIC energy range from $23 \sim \mathrm{GeV} / \mathrm{c}$ to $250 \sim \mathrm{GeV} / \mathrm{c}$. The sizable analyzing power, the large cross section and the advantages of a solid ribbon target makes this process ideal for a fast primary polarimeter for RHIC.

A typical $(-t)$ value of 0.002 to $0.01 \mathrm{GeV}^{2}$ results at high energy in a very small angle of the forward scattered proton relative to the beam direction and also a very low kinetic energy of the Carbon recoil of about $0.1-1 \mathrm{MeV}$. It will be impossible to measure the forward scattered proton without drastically reducing the beam divergence at the target, which would severely reduce the scattering rate and also cause unacceptable beam emittance growth. It will therefore be necessary to rely only on the measurement of the recoil Carbon nucleus to identify elastic scattering.

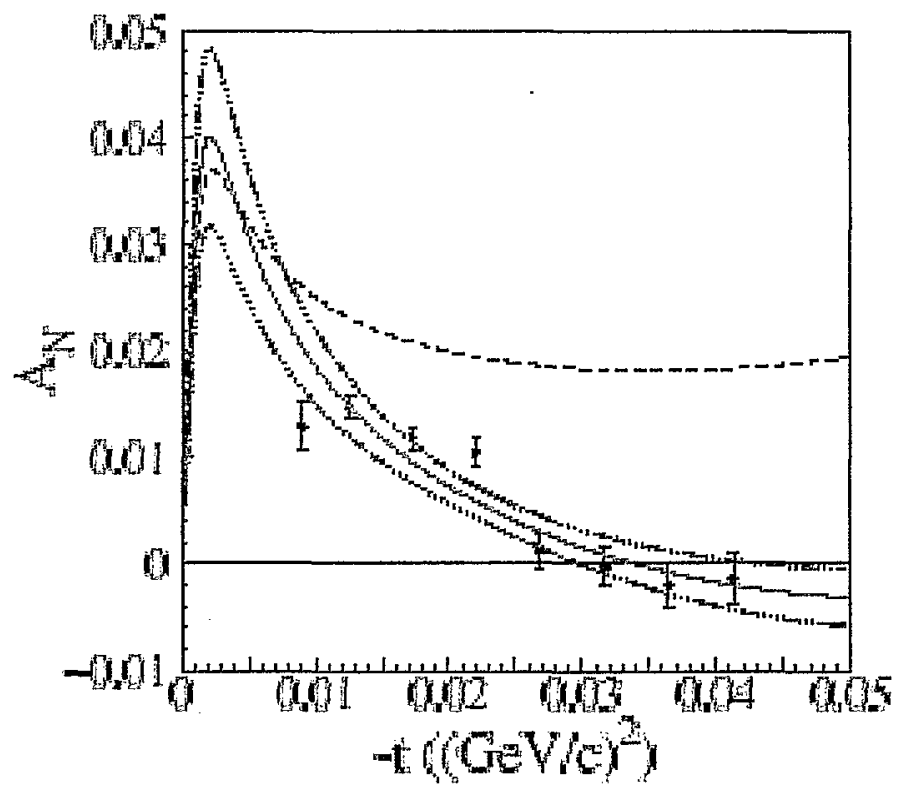

FIGURE 2. The analyzing power, $A_{N}$, for $p C$ elastic scattering in the CNI region. The error bars on the data points are statistical only. The solid line is the fitted functions from theory. The dotted lines are the 1-sigma error band of the fitting results. The dashed line is the theoretical function with no hadronic spin-flip amplitude.

Direct measurement of the 0.1 to $1 \mathrm{MeV}$ recoil Carbon nuclei is only possible for a very thin Carbon target. The detector for the recoil carbon must be compact with detectors at about $20 \mathrm{~cm}$ away and in the beam vacuum. The distance has to be far enough to gate out the fast prompt particles but close enough for the slow carbon particles to reach the detectors before next bunch comes. The detectors are silicon strip detector (SSD) with sizes of the order of centimeters. Very thin micro-ribbon carbon targets assure the recoil carbons to exit the target. Carbon target is the preferred 
target but the possibility that a nuclear target might present a large dilution of the observed asymmetries was a concern.

To test the idea, a proof of principle experiment was conducted in the AGS in 1999. The AGS Experiment E950 used AGS polarized proton beam to measure the asymmetry in p-C CNI process at the RHIC injection energy around $23 \mathrm{GeV}$. The experiments also studied count rate limitations and problems in identifying elastic scattering events by using the energy, time of flight, and angle information measured with the recoil detector alone. The experimental results show that the recoil carbons from CNI scattering can be obtained with the Si detector and the asymmetry behavior follows the theory qualitatively ${ }^{3}$. Given the success of AGS test, we have decided to develop a p-Carbon CNI polarimeter for RHIC.

\section{THE DETECTOR DESIGN}

In this section, the design of the detector and the readout scheme are described. Due to the fact that it is impossible to detect scattered beam protons, the elastic scattering events have to be identified by only detecting recoiled Carbons. The system needs to allow measurements of Carbon arrival timing with respect to the beam bunch crossing (RF clock) as well as the Carbon kinetic energy to improve the signal to noise ratio. The distance of the detector is adjusted so that even the lowest energy Carbons $(\sim 100 \mathrm{keV})$ can arrive before the next RHIC bunch passes the target. The bunchcrossing interval is typically $106 \mathrm{~ns}$ at RHIC. The late arrival time of the recoiled Carbons is advantageous since the detection can be done in almost noise-free environment after all beam-induced disturbances are gone.

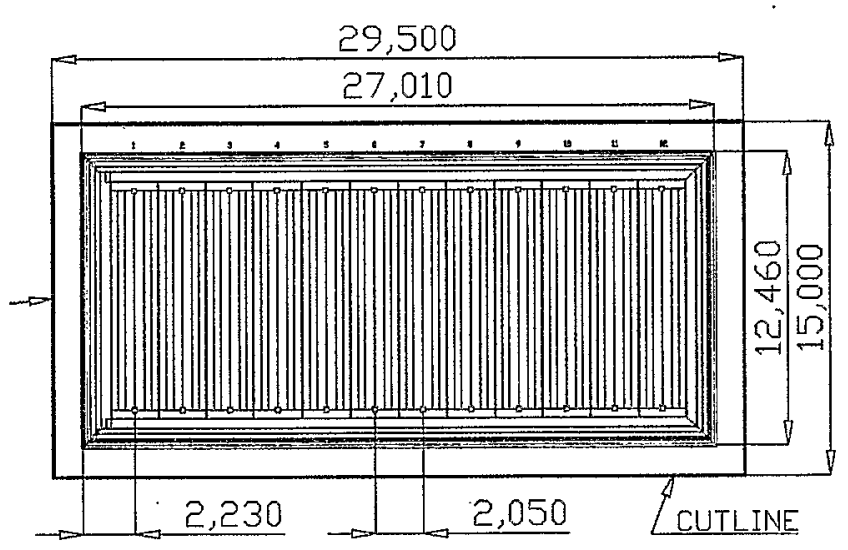

FIGURE 3. Si strip detector (SSD) design. Twelve strips consist of the sensitive area and are surrounded by five guard rings. The scale unit is micrometer.

\section{Si Detector}

We chose Si strip detector for recoiled Carbon detection since it is the only device, which allows measurements of energy and arrival time of Carbons in the RHIC ring vacuum. environment. The detection inside the vacuum is necessary because the 
recoiled Carbon energy as low as $100 \mathrm{keV}$ cannot penetrate even the thinnest vacuum window available.

The Si detector group in the instrumentation division of Brookhaven National Laboratory developed a thin window Si strip detector. Figure 3 shows the sensor front surface design. The size of the active area of the detector is about $10 \times 25 \mathrm{~mm}^{2}$. The size was chosen to cover the CNI kinematical region for the recoiled Carbons. The active area is divided into 12 strips and each strip has $10 \times 2 \mathrm{~mm}^{2}$ size which was determined to realize low noise operation since the pickup noise increases with $\sqrt{C}$ where $C$ is the capacitance of a detector channel. The typical capacitance of a strip at full depletion is $6 \mathrm{pF}$.

For the detection of low energy Carbons, dead layer of Si sensor is undesirable but cannot be avoided during the production. The energy and the density of the Boron implantation was optimized to have the thinnest dead layer to allow low energy Carbon detection. $\mathrm{SiO}_{2}$ protection layer, which is developed during the course of the implantation procedure, is normally left to protect the surface of the sensor for stable yield but is removed for our purpose. The dead layer thickness is expected to be 25-40 $\mu \mathrm{g} / \mathrm{cm}^{2}$.

For the installation of the Si sensor, there are two requirements. One is that the distance between the sensor and the preamplifier has to be short for a good signal to noise ratio. The other is that the sensor assembly needs to be compatible with the RHIC ultrahigh vacuum $\left(10^{-9}-10^{-10}\right.$ Torr). To fulfill both requirements, Si sensor mount is separated from preamplifier board and is installed inside the vacuum on two 15-pin-D-subminiature feedthrough connectors. The preamplifier boards are directly coupled on the other side of the vacuum feedthrough. In case of baking, the preamplifier boards can be removed from the vacuum chamber.

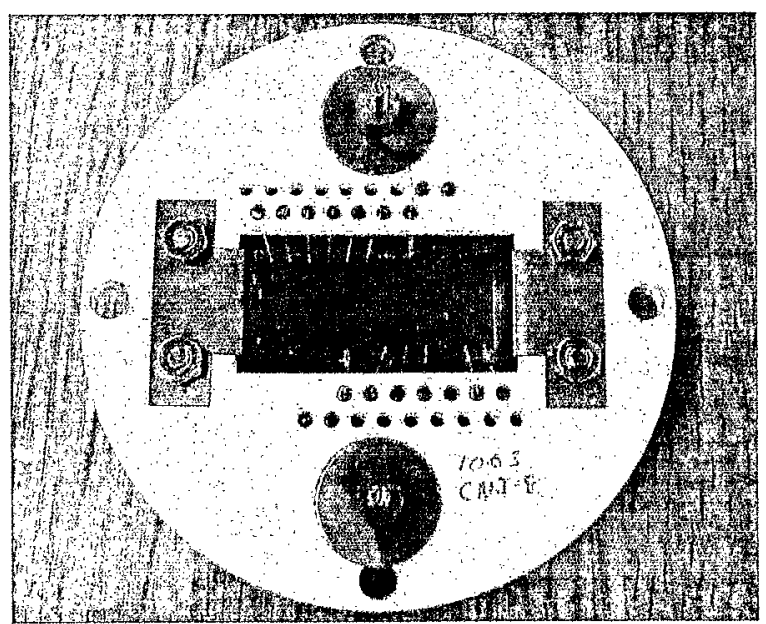

FIGURE 4. The Si mount assembly.

The Si mount consists of $\mathrm{MACOR}^{\odot}$ disks, Be-Cu plates and split pins. A Si sensor is mounted on the disk with two plate springs. The split pins are held in position by sandwiching their shoulders between two $\mathrm{MACOR}^{\mathbb{G}}$ disks. They are aligned such that the rows match with a 15-pin-D-subminiature connector. The electrical connections are made by wire bonding each Si strips and the head of the split pins. 
From the experience in AGS E950, the angle information is not essential; the strips will be mounted parallel to the beam direction in RHIC. The event rate of each strip will only be one twelfth of rate of the total detector.

\section{Preamplifier and Shaper}

The preamplifier boards are mounted on the polarimeter vacuum chamber directly connected to the $\mathrm{D}$-subminiature feedthrough to minimize the distance to the signal source. The preamplifier used is a modified version of IO446-2 made by the instrumentation division of Brookhaven National Laboratory. Due to the fact that the timing resolution is more important than the energy resolution in terms of determining the kinetic energy of Carbons, the rise time is minimized and the decay time was shortened to $50 \mu \mathrm{s}$ for high rate capability.

The pre-amplified analog signals are transferred through the RHIC tunnel on coaxial cables (RG59) for about 300 feet to a counting room located next to the tunnel. The signal process outside the tunnel is necessary because extensive debugging is essential for commissioning a new device while the access is very much limited in a collider accelerator. Commonly available RG59 (75 $\Omega$ ) was chosen over RG58 (50 $\Omega$ ) because the signal attenuation was found to be slightly smaller on a test bench.

The analog signals are fed into hybrid shaper amplifiers; IO532-01 designed by the Brookhaven instrumentation division group. The shaping time is kept at $12 \mathrm{~ns}$ from the original design for optimization of the timing resolution. Its original bipolar output was modified to unipolar because the following readout electronics (Wave Form Digitizer) has an input range between 0 and $1 \mathrm{~V}$. The Si sensor readout scheme is shown in figure 5.

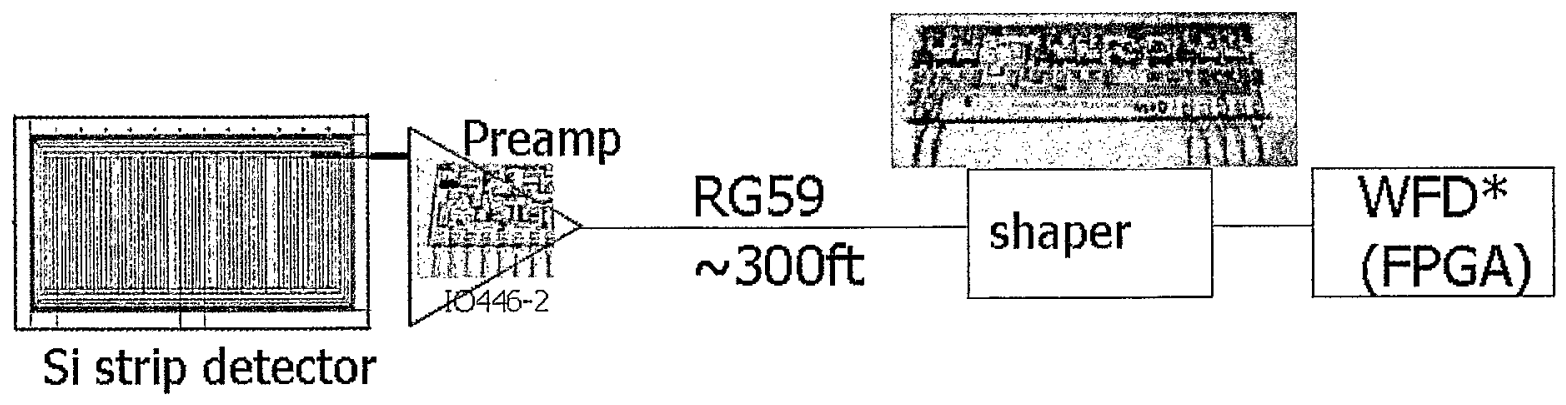

FIGURE 5. The Si sensor readout scheme.

\section{WAVE FORM DIGITIZER}

Although the absolute value of the analyzing power of $p C$ CNI scattering is small, the cross section is fairly large which allows a measurement of statistically significant asymmetry within half a minute. The event rate per strip is estimated to be on the order of $100 \mathrm{KHz}$ for normal RHIC running condition. It is not trivial to handle this high event rate with conventional charge sensitive ADC such as $2228 \mathrm{~W}$ or FERA ADC of LeCroy Corporation. Another problem with those conventional ADCs is the 
dead time. In principle, dead time can be corrected with careful calibrations but sizable systematic errors are left after applying the corrections in practice.

The Wave Form Digitizer (WFD), which was originally developed for g-2 experiment at BNL by Yale University, was one of the solutions, which solve the both problems. The Yale WFD technique was adopted for the CNI polarimeter readout.

\section{Hardware}

Figure 6 shows the graphical description of the WFD design. It is generally a good practice to accommodate commonly available electronic devices for a research project. It avoids the necessity of developing customized electronics and leads to reduction of the development time and cost. The Yale WFD is one of such examples. It utilizes a laptop computer LCD driver 8-bit-ADC. It is designed to keep digitizing the display analog signal, which is continuously fed from the CPU at the rate of $140 \mathrm{MHz}$. The ADC has three channels corresponding to RGB. Since no color information is necessary for the CNI polarimeter readout, each input is split into three and sent to the three ADC channels. Two out of the three transmission lines have longer traces on the PC board and they are delayed by one third and two thirds of the digitizing cycle respectively. This arrangement makes the three-channel-140Mhz-ADC work as a $420 \mathrm{MHz}$ ADC. The relative delays are realized on the printed traces.

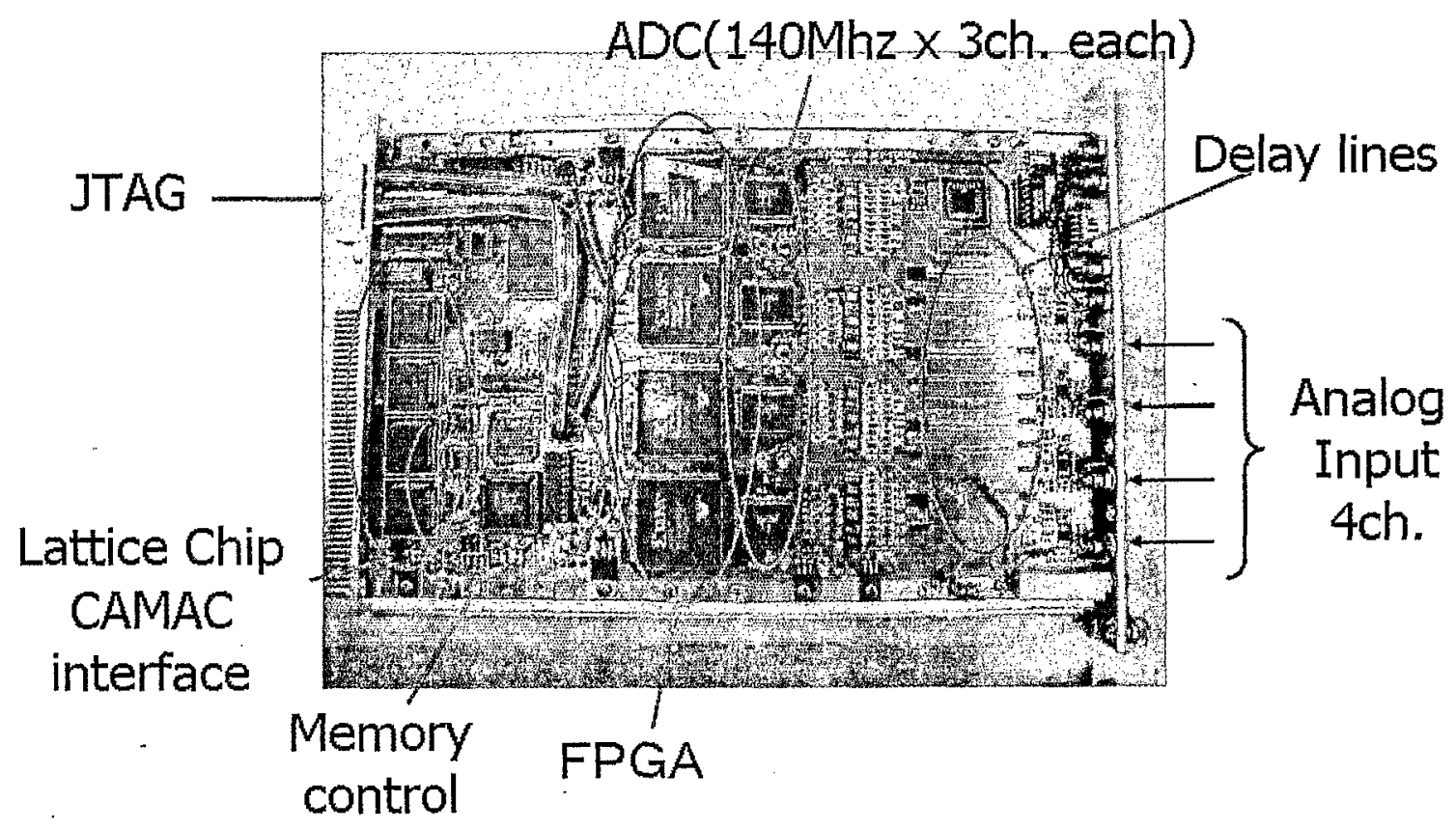

FIGURE 6. The WFD design of the RHIC CNI polarimeter.

After analog signals are digitized, all three channel $A D C$ values are sent to one Xilinx FPGA and data processing is done with the following FPGA algorithms. DRAM is implemented on the board using Xilinx utility for large data storage. Interface to the CAMAC is realized using Lattice PLD chips. External JTAG connector is also available for downloading programs to those programmable devices. 


\section{FPGA Algorithm}

There are basically three readout modes made available by the FPGA code developed for the CNI polarimeter. They are namely (1) wave form mode, (2) histogram mode and (3) scaler mode.

In the waveform mode, FPGA program basically resorts the digitized values, which pass the preset threshold into the right time sequence. It sends the data to the memory section along with the bunch crossing number and the polarization information for storage. The analysis is done offline after all the data taking is done. If the wave form of the previous bunch does not exceed the threshold, the code defines the flat level to be the ground and the subtraction from the current wave form is always done. This feature alleviates the effect of the baseline fluctuation which often results in deteriorated quality of the data.

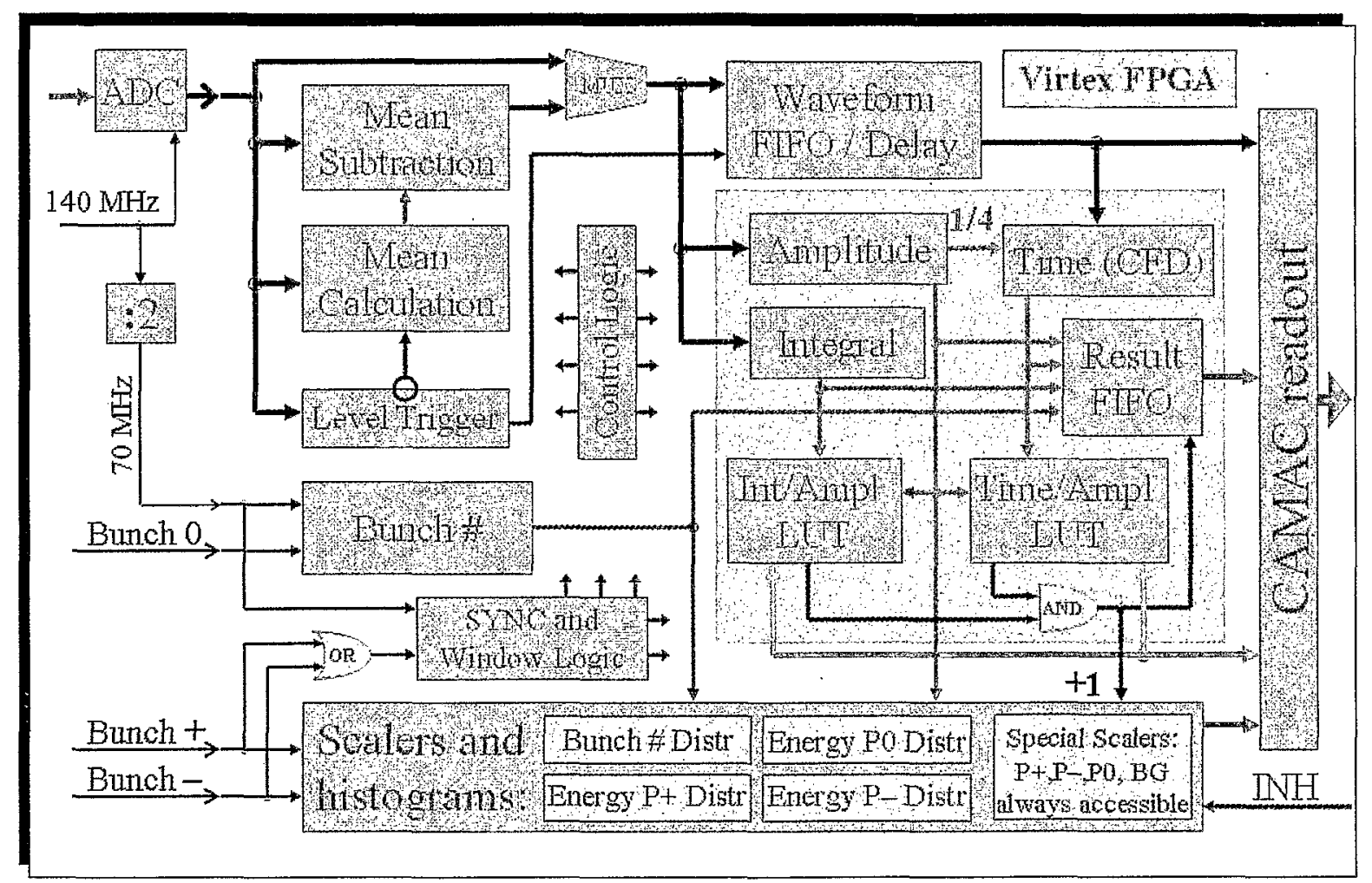

FIGURE 7. The block diagram of the WFD FPGA code algorithm.

The FPGA code extracts pulse height and the arrival time from waveforms, which pass the preset threshold and store them along with the bunch number and polarization information in the histogram mode. The pulse height is defined as the maximum of the digitized values within the pre-determined time-window with respect to the bunchcrossing signal. Once the maximum is found, the timing search algorithm follows the waveform backward in time. When the waveform becomes less than one forth of the maximum, the code determines the corresponding time bin as the arrival time of the pulse. Therefore, it serves as a constant fraction discriminator. 
Lastly the scaler mode extracts the Carbon bands and counts them according to the bunch crossing signal and the polarization information. The determination of the Carbon band is done by pre-loaded bin-by-bin maximum and minimum of time limits are used. This is the mode commonly used for normal operation during the physics running period.

\section{CHAMBER DES IGN}

The inclusive pion polarimeter was originally considered for $\mathrm{RHIC}^{4}$ and a long straight section is needed. For easy maintenance, this has to be a warm straight section. The warm straight section between Q3 (quadrupole 3) and Q4 (quadrupole 4) at section 12 is assigned for both polarimeters in blue and yellow rings. Both target chambers are located near Q4 where the vertical and horizontal lattice $\beta$ functions are small thus reducing the effect of multiple scattering on emittance dilution. Both target chambers are upstream of each other. Since beams are in opposite directions in the two rings, the detectors for both polarimeters are not in the scattering showers of each other. The target chamber for yellow ring is also close to Q4, which gives enough space for the one-arm inclusive pion polarimeter (sharing the same target), which was designed as a back up polarimeter but never needed.

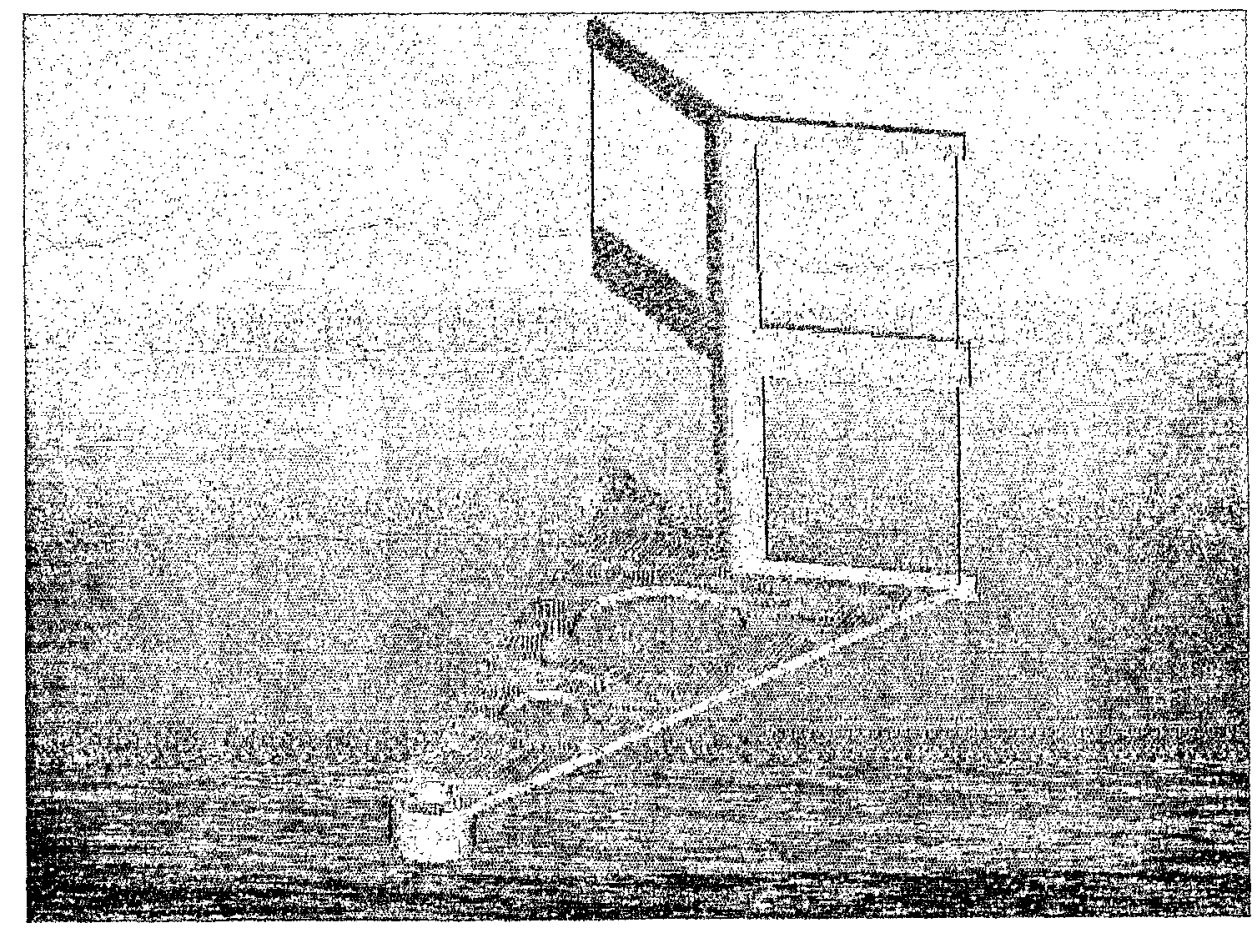

FIGURE 8. Metal target frame and the ceramic $V$ Plate. The holes in the $V$ shape reduce the inertia of the target assembly. The target is a $6-8 \mu \mathrm{m}$ wide, $4 \mu \mathrm{g} / \mathrm{cm}^{2}$ ultra-thin carbon ribbon. It is $3 \mathrm{~cm}$ long.

It is desirable for the polarimeter to measure both horizontal and vertical beam polarization profiles, which requires separate targets scanning both vertically and horizontally. Since the thin carbon target has a relative short lifetime at the full RHIC beam luminosity, it would be advantageous to mount more than one ribbon on the 
driving mechanism (spaced so that the beam sees one ribbon at a time) to provide redundancy in case of ribbon breakage. The original design of target holder is capable of holding 4 targets, as shown in Figure 8. Each target assembly has two motions: linear motion to move whole assembly in and rotary motion to move the desired target into the beam. An interlock relay system is in place to assure that at any time only one of the two assemblies can move into the beam.

To reduce the impact on beam impedance, the radius of the box should be as close as possible to that of the beam pipe, i.e., $6.5 \mathrm{~cm}^{5}$. The target box radius is $16.03 \mathrm{~cm}$, which is minimum to host the target driving assembly.

In manipulating polarization in RHIC, information on both vertical and horizontal components of beam polarization is needed. In addition to one pair of detector sitting in the horizontal plane, two pairs of detectors sitting at $45^{\circ}$ are added, which are capable of measuring both transverse components. When using these two pairs for vertical components, the analyzing power will drop by a factor of $\cos 45^{\circ}=\sqrt{2} / 2$. At full RHIC designed intensity, the bunch width is about 2 ns and bunch spacing is $106 \mathrm{~ns}$. To avoid the prompt background, the carbon nuclei should arrive the detectors between two bunches, i.e., within 100ns. The Si detector can detect carbons with kinetic energy as low as $200 \mathrm{keV}$, which can travel about $20 \mathrm{~cm}$ in $100 \mathrm{~ns}$. The distance between detectors and interaction point is set as $20 \mathrm{~cm}$. This is slightly more than the radius of the chamber. There are RF shields at the surface of the chamber to cover the detector ports, which reduce the impedance impact.

The minimum target box length is determined by the relative position of target, view port and detector sockets, which is $50.8 \mathrm{~cm}$. Due to the impedance constraint, two 5:1 transition sections are attached at both sides and give the total length of the target chamber as $1.6 \mathrm{~m}$. The top view port is used for target installation and target position monitoring. Total of six silicon strip detectors are used. They are upstream of the target assemblies so there is less background due to forwarding scattering particles from targets. Due to the complicated shape of the target assemblies, the detector at horizontal plane on the driving motor side is partially blocked by the ceramic piece of the horizontal moving targets. So the $45^{\circ}$. detectors will have to be used for these targets, which will be used for vertical beam polarization profile scan. On the other hand, the vertical moving targets are cleared for all detectors. The vertical moving targets should be the primary targets.

The relatively high intensity and short bunch proton beam in RHIC can generate wake fields inside the beam components. The long-range wake field is more harmful for RHIC longitudinal beam dynamics. In other words, without a lowimpedance component design, the proton beam could lose more energy in the component. The long-range wake potential in time domain corresponds to the narrowband impedance in frequency domain. The shunt impedance of RHIC beam components must be kept below certain limits to prevent emittance blowup and possible beam loss due to coupled bunch instabilities. Consequently, the RHIC CNI polarimeter target box requires a careful impedance analysis to fit into RHIC impedance budget. During conceptual design stage, we used a 3-D electromagnetic field codes MAFIA to minimize the shunt impedance of structure. It shows that shunt impedances of the target box for all target positions are below the impedance limits. 
With 5:1 tapers and insulated target design, the shunt impedance of RHIC polarimeter is under impedance limits of both injection and storage cavities. The 3-D plots of the inside and outside of the target box are shown in Figures 9-10.

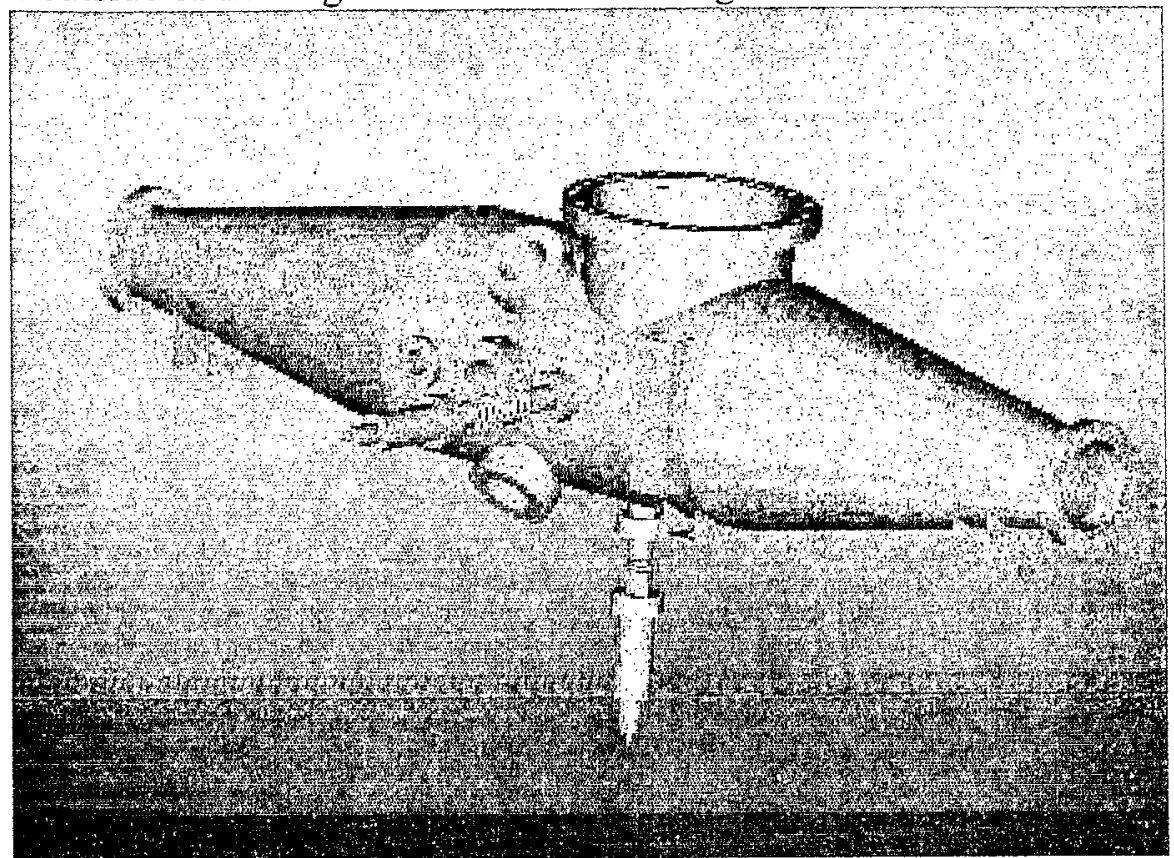

FIGURE 9. RHIC CNI polarimeter target box. Assembly view of the target chamber. Beam goes from the left to the right.

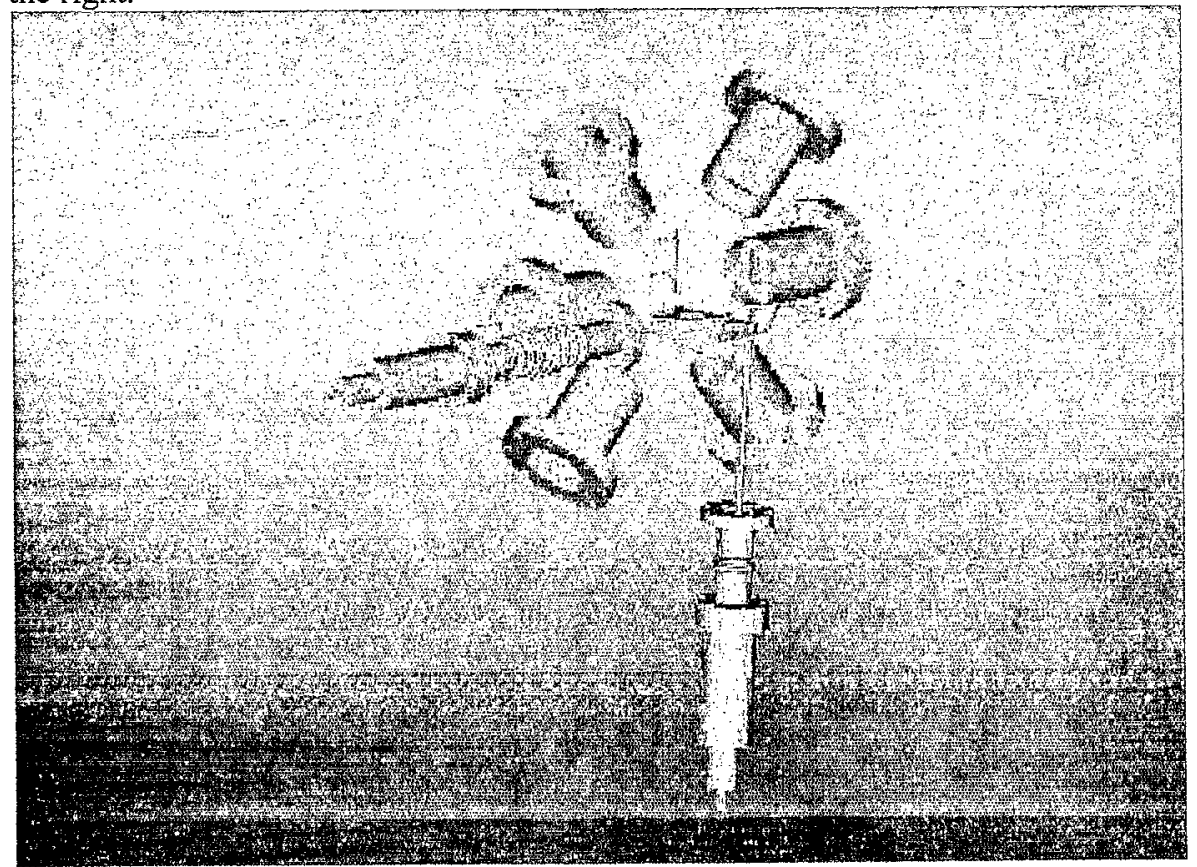

FIGURE 10. Exploded view of RHIC polarimeter target chamber, it shows the relative positions of the six Si detectors and two target holders. An interlock relay box is used to prevent the clash of the two target holders. 


\section{CARBON RIBBON TARGET}

Carbon target played an important role in deciding what process to be used for RHIC and AGS polarimetry. The production of the carbon ribbon targets has been developed at IUCF, and now is routine. We chose $p C$ CNI over $p p$ CNI for the RHIC polarimetry because and the ultra-thin carbon ribbon was available, which would allow the low energy carbon recoils to escape the target. The target would survive heating from the RHIC beam, provide sufficient luminosity for a quick precise polarization measurement, be sufficiently thin o avoid pile-up of events in the detector at the same time. For a thicker target, only surface scattering would produce observable recoils in the CNI region, and the target would fail on the other points as well (heating, pile-up). A hydrocarbon target, for pp CNI, would not survive both RHIC and AGS beam. A polarized jet target, which has been used for absolute polarization calibration, is technically difficult and not enough rate to get fast measurement.

The ribbon is mounted perpendicular to the beam direction in free suspension between the ends of a target frame. The target frame is then installed onto a target holder, which can hold four or six targets. Such a ribbon mounted on target frame is shown in Figure 11.

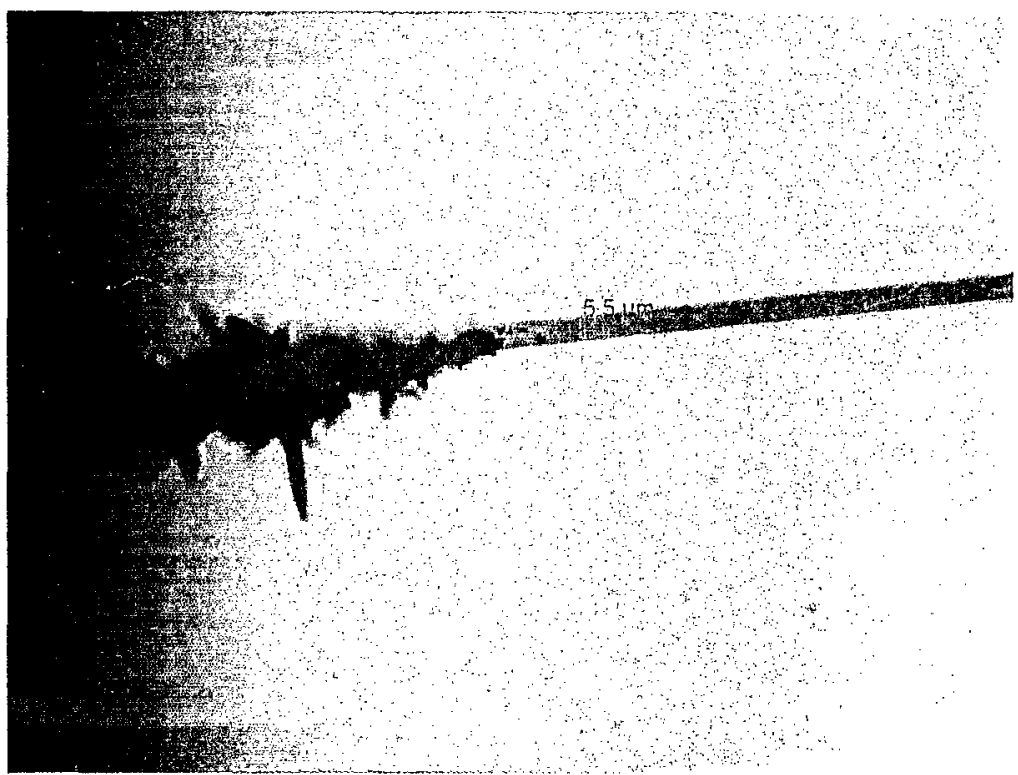

FIGURE 11. This is the RHIC target under microscope (500 times). Left dark part is the target frame. A lump of blue can be seen between the target ribbon and the frame. The size of the target ribbon is about $5.5 \mu \mathrm{m}$.

The survival of the target under RHIC intense beam is crucial. Of the total ionization energy loss of the protons interacting with the carbon fiber, $30 \%$ is deposited on the target in the form of heat ${ }^{6}$. Figure 12 shows the expected equilibrium temperature as a function of beam energy for a typical carbon ribbon target. It is generally inadvisable to run the fiber at temperatures exceeding $2000 \mathrm{~K}$, which is the onset of thermionic emission, since this would shorten the lifetime of the fiber. The result shows, with the ribbon target, heating is not a problem even with smaller beam emittance $\varepsilon_{\mathrm{N}}=10 \pi \mu \mathrm{mrad}$. 


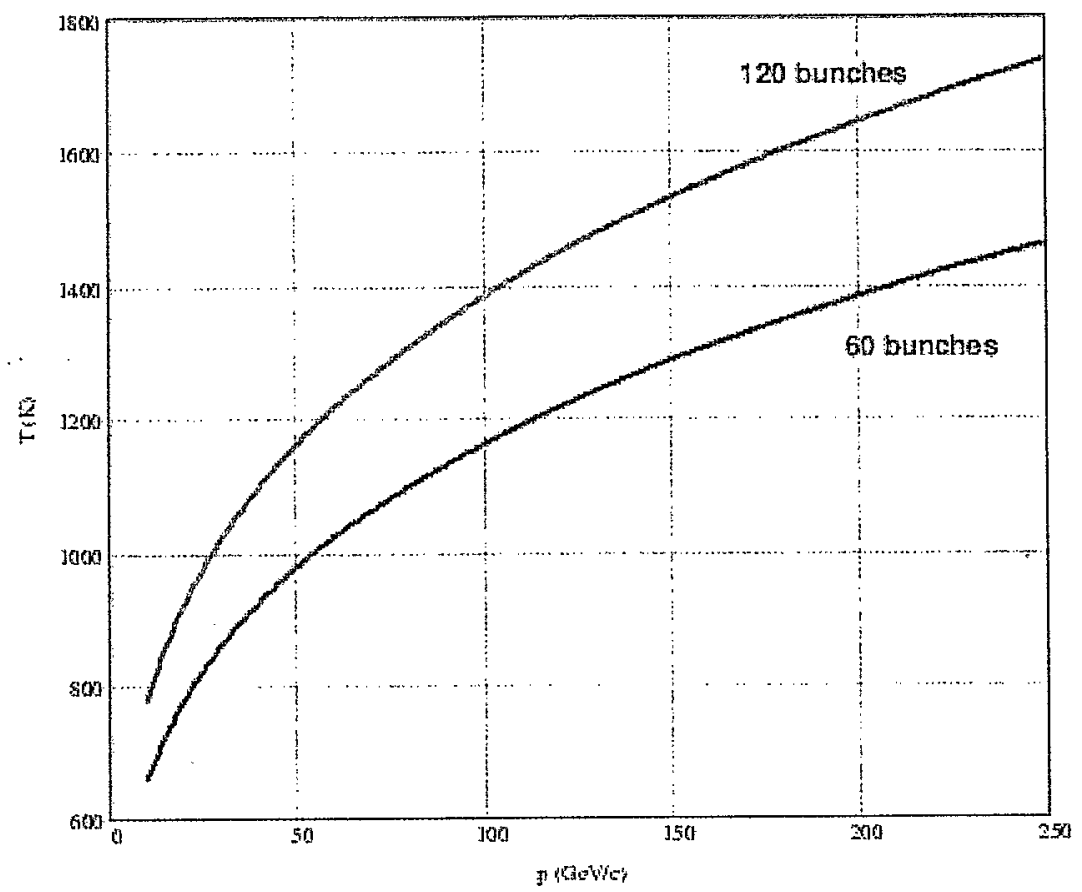

FIGURE 12. Equilibrium temperature of a $4 \mu \mathrm{g} / \mathrm{cm}^{2}$ carbon ribbon for two beam currents ( 60 bunches and 120 bunches). The beam parameters are: $2 * 10^{11}$ protons per bunch, $\varepsilon_{\mathrm{N}}=10 \pi \mu \mathrm{mrad}$. In real operation, there has to be bunch gaps for abort kicker working properly. The maximum number of bunches is 112 .

\section{OPERATION}

Figure 13 shows the polarimeters installed in RHIC. Typical RHIC physics store is 8-10 hours. Polarization is measured in both rings at the beginning of the store and every 2-3 hours. Past experience shows that there is some gradual polarization loss over the store. The loss is driven by some high order depolarizing resonances, which are very weak but do show effect in the long time scale. Since every polarization measurement causes some beam loss $(<0.5 \%)$ and some emittance growth, we compromise to measure polarization every two to three hours. 


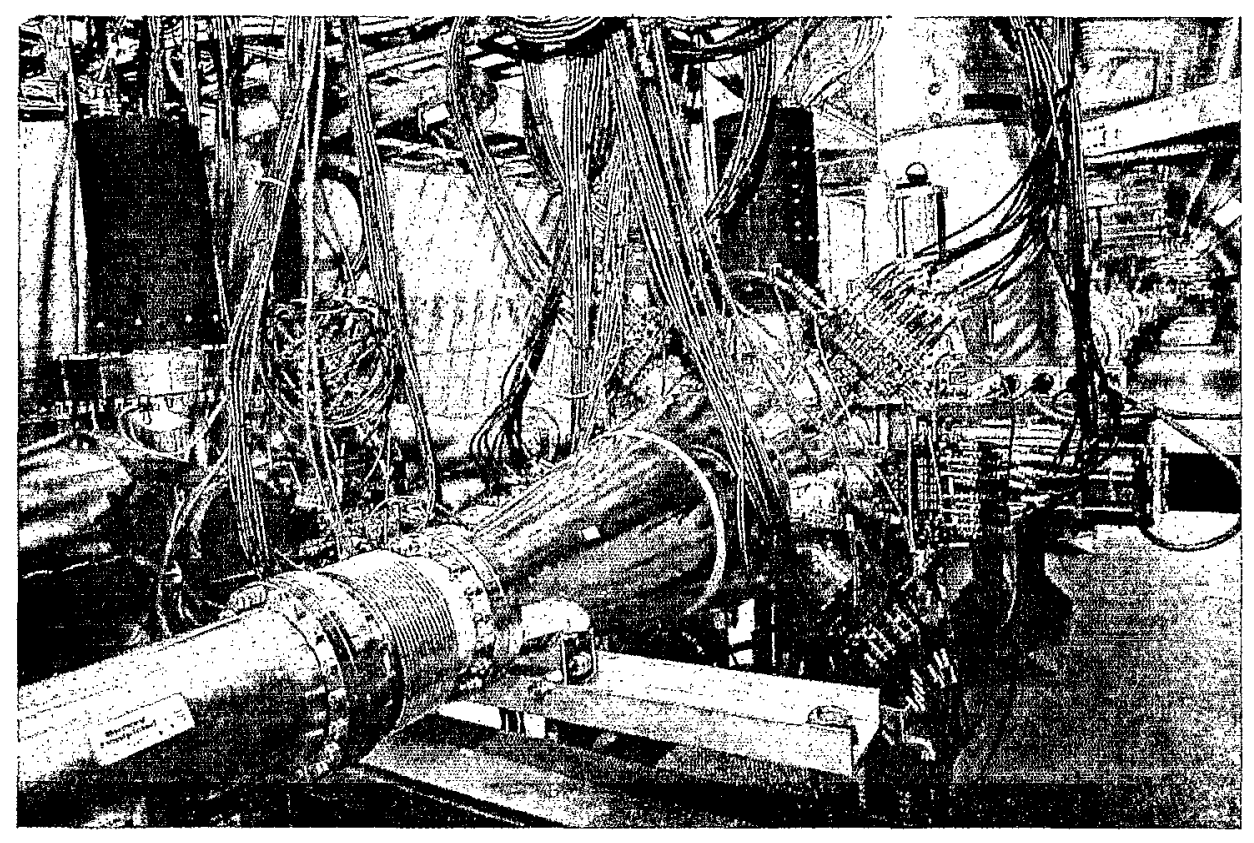

FIGURE 13. The RHIC polarimeters in RHIC tunnel. The front one is polarimeter in blue ring and the one behind it on the left is the polarimeter in yellow ring. Six detectors surrounded the chamber. The horizontal target drive motors are visible for the blue polarimeter.

A polarimeter control application has been developed over years and non-experts use it. It consists of target scan page, polarization measurement page and data plot page. The example of the application is shown in Figure 14. One can select the target (between 1 and 6), orientation (horizontal or vertical) from the page. The measurement can be either a fixed target position measurement or a profile measurement with target scanning through the beam. The beam center position can be determined from a target scan (scanning target through beam). For regular operation when the target, total number events and measurement mode are already determined, the measurement is just a click of the run button. The carbon target will be moved in during the measurement and pulled out after the measurement. The polarization value is updated seconds after the measurement. The polarization plot page is used to judge the quality of the measurement and is used by operators daily. An example is shown in Figure 15. 


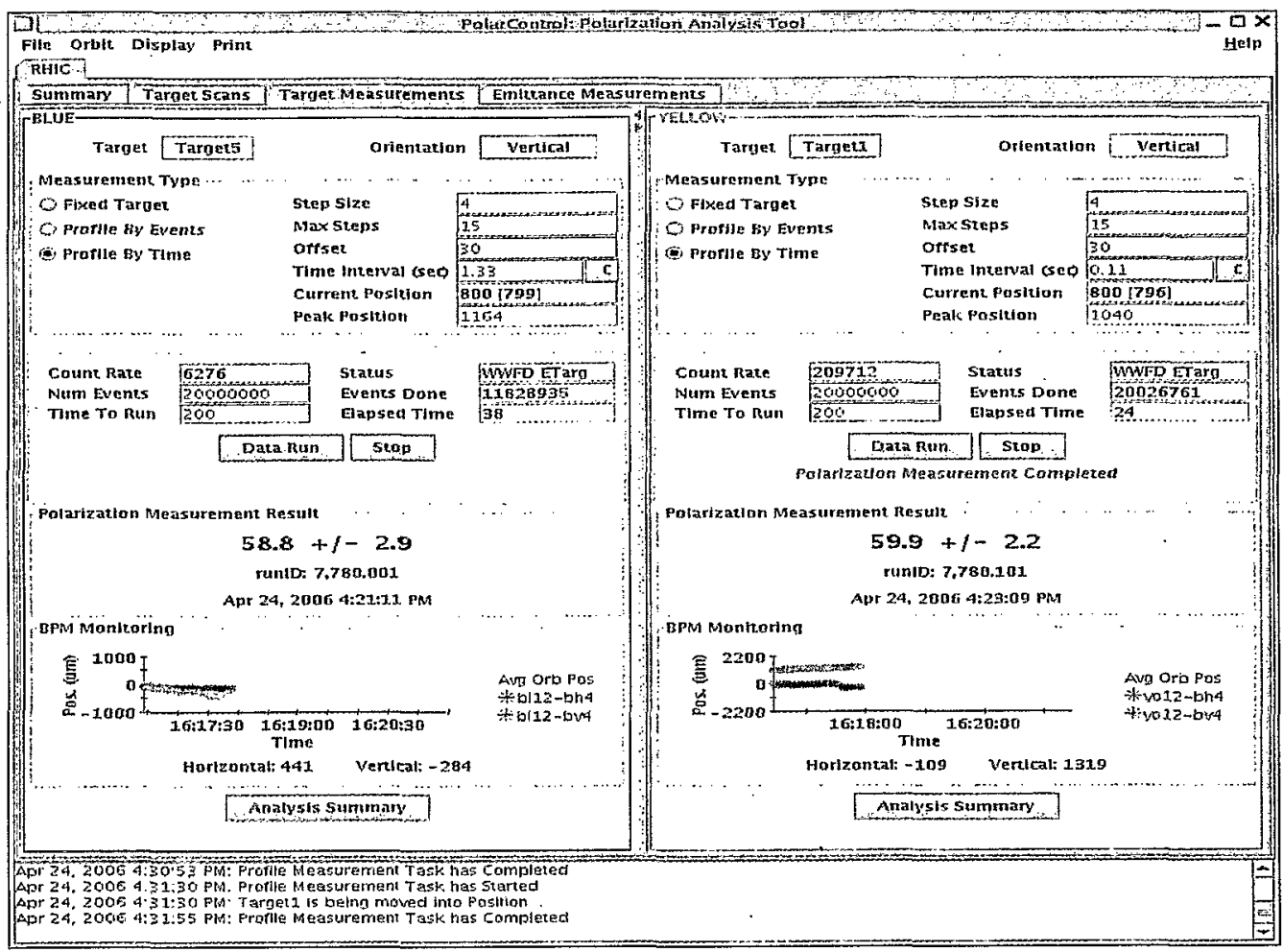

FIGURE 14. Polarimeter application page. The bottom plots are the BPMs near the polarimeter targets. They are used to determine the desired target positions.

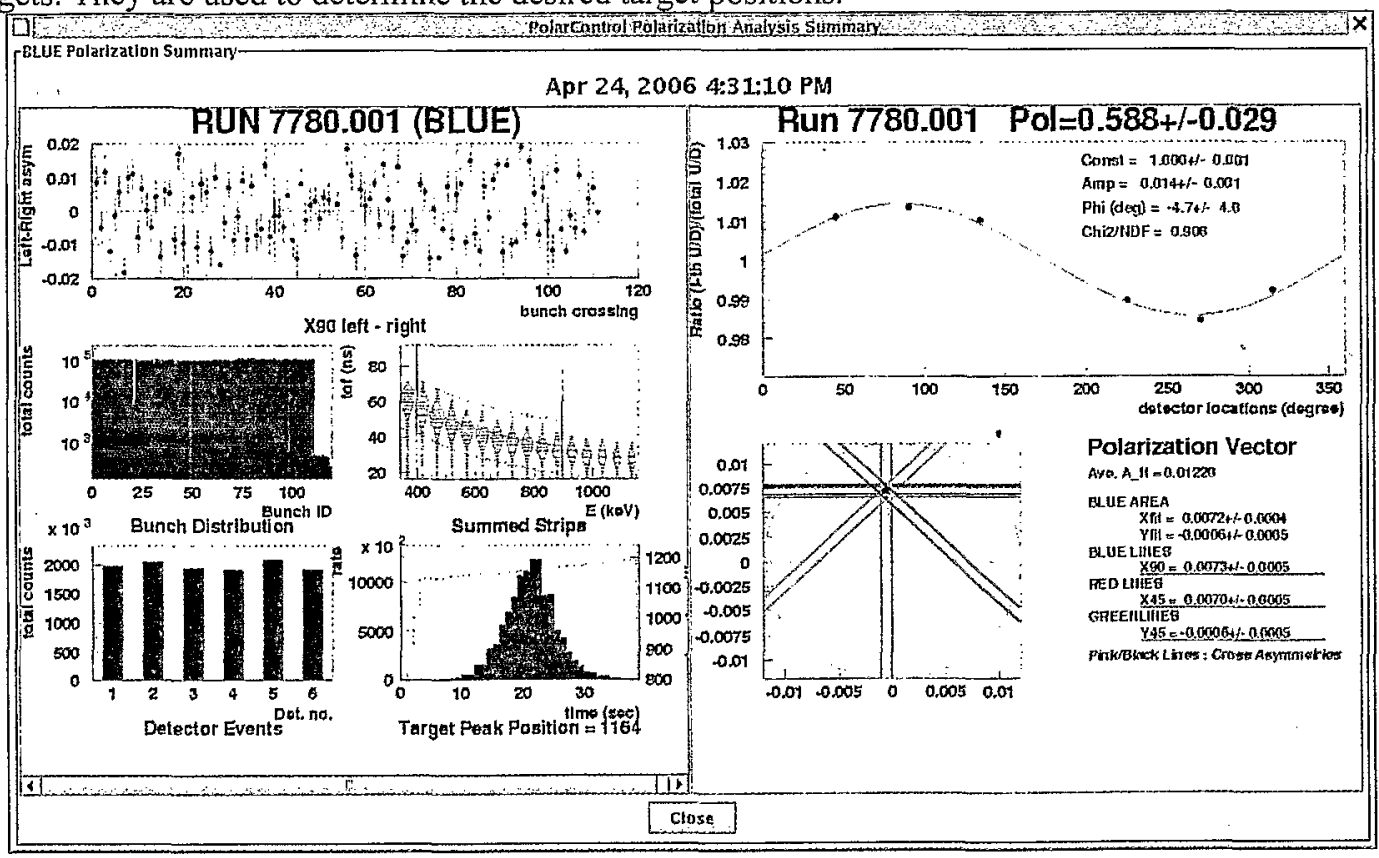

FIGURE 15. Example of a polarization plots for blue ring. The various plots can be used to monitor the behavior of the polarimeter.

With WFDs, every elastic event has a time stamp attached. We can tell the event comes from which bunch crossing. In principle, with the target scan through the beam, one can get bunch-by-bunch emittance information. A software application has been 
developed to implement the idea. Figure 16 'shows an example of an emittance measurement in blue ring.

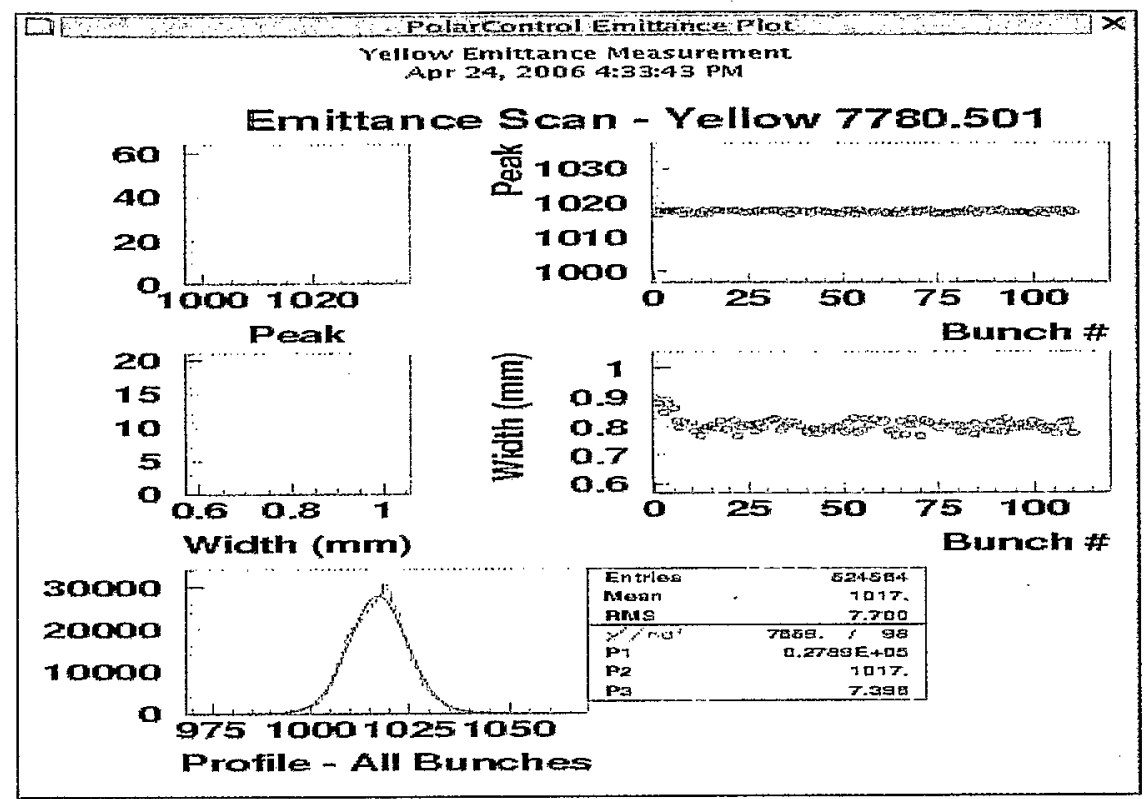

FIGURE 16. Emittance measurement in Yellow ring. The profile at left bottom is for all 111 bunches, the beam width in the middle is for all individual bunches.

Over the years, there have been great improvements in RHIC luminosity and polarization. As shown in the following table, in past five years, the bunch intensity and number of bunches have been doubled. The polarization has increased four times. Polarimeter played a critical role in accelerator setup for higher polarization.

\begin{tabular}{|l|c|c|c|c|c|}
\hline & 2002 & 2003 & 2004 & 2005 & 2006 \\
\hline Bunch Intensity $\left(10^{11}\right)$ & 0.7 & 0.7 & 0.7 & 0.9 & 1.4 \\
\hline Number of Bunches & 55 & 55 & 56 & 109 & 111 \\
\hline Polarization & $15 \%$ & $35 \%$ & $46 \%$ & $47 \%$ & $\sim 65 \%$ \\
\hline
\end{tabular}

In these years's operation, there are also many problems encountered and solved. Due to the limited acceptance of the detectors, the target mounting and installation have to provide accuracy of $+-0.5 \mathrm{~mm}$. This requires control of target mounting on the target frame and installation of target frame onto target holder. All targets are surveyed after installation in the ring to make sure we have targets at the desired location. Third, production of desired target size.

The Si detector leakage current increases with radiation dose. Since Si detectors are installed inside the chamber, they are very sensitive to any local beam loss. We have read backs of all detector leakage currents now. The plan for future operation is to turn them on only during physics store. They will remain off during injection, ramp and beam dump.

Due to the limited lifetime of carbon targets and Si detectors, the polarimeter chambers have to be open every year before polarized proton run starts to replace the 
targets and detectors. In addition, target replacement during a run is also required as beam intensity increased over years. Due to the radiation damage of beam, the target has limited lifetime, which is about two weeks on average with intensity of $1.4^{*} 10^{13}$. Experimenters would prefer to use vertical targets to get polarization measurements. Even though we changed the target holder to increase the number of vertical targets in one installation to six, it is still required a target replacement during run. This means interruption of RHIC operation and bleed up vacuum. A fast turn around time is crucial for RHIC running efficiency. Due to the complexity of the chamber and the electronics, it is not practical to bake the chamber. Even if it is baked, the benefit is lost after opening. First effort is to eliminate the high out-gassing component from the chamber. We carefully measured all components' out gassing rates. The high out gassing rate ceramic material is replaced with low out-gassing rate material. A NEG (non-evaporable getter) cartridge pump is added to each chamber to provide additional continuous pumping. As a result, the pump down speed is greatly improved. A full intensity physics store can be resumed within 24 hours (vacuum down to $10^{-9}$ Torr).

\section{SUMMARY}

A new kind of relative polarimeter, which measures proton-Carbon elastic scattering at small angles (CNI region), has been implemented in RHIC and AGS successfully. This polarimeter is ideal for high-energy proton polarimetry: fast measurement, low cost and compact size. The $A_{N}$ of the pC CNI polarimeter has been calibrated by a polarized jet at $100 \mathrm{GeV}$. The jet continues to provide such a calibration for current run.

\section{ACKNOWLEDGMENTS}

The authors would like to thank many people, physicists and engineers who have worked on and are working on both AGS and RHIC CNI polarimeters: I. Alekseev, A. Bravar, G. Bunce, T. Curcio A. Deshpande, S. Dhawan, D. Fields, D. Gassner, R. Gill, D. Hseuh, G. Igo, K. Imai, O. Jinnouchi, V. Kanavets, D. Lehn, Z. Li, G. Mahler, Y. Makdisi, I. Nakagawa, W. Lozowski, W.W. MacKay, C. Naylor, B. Oerter, S. Rescia, T. Roser, T. Russo J. Saetta, N. Saito, H. Spinka, D. Steski, D. Svirida, Y. Tian, R. Todd, J. Tojo, D. Underwood, C. Witten, J. Wood.

\section{REFERENCES}

1. W.R. Lozowski and J.D. Hudson, NIM in Physics Research A334, 173(1993).

2.N.H. Buttimore et al., Phys. Rev. D 18, 694(1978); N.H. Buttimore, AIP Conf. Proc.95,(AIP, New York, 1983), p. 634.

3. J. Tojo, et al, Phys. Rev. Lett. 89, 052302(2002).

4. I. Alekseev, et a1, Design Manual of Polarized Proton Collider at RHIC, July, 1998, unpublished.

5. H. Wang and H. Huang, RHIC Polarimeter Impedance Analysis with MAFIA, RHIC/AP/178(October, 1999), unpublished. 
6. J.L. Rosen, AIP Conf. Proc.26,(AIP, New York, 1975), p. 287. 